\title{
Grain boundary component in W-Ga composites: a way towards skeleton structures
}

\author{
W. Krauss and H. Gleiter* \\ Technical Physics, University of Saarbrücken, D-66041 Saarbrücken, Germany * Forschungszentrum Karlsruhe GmbH, \\ Postfach 3640, D-76021 Karlsruhe
}

(October 18, 2018)

\begin{abstract}
Nanostructured materials consist of crystalline and grain boundary components. In the simplest case, both components are chemically identical. Here, we present the results of a study of a system consisting of a crystalline component built by $\mathrm{A}$ atoms (tungsten) and a grain boundary component of B atoms (gallium). Within this system, component B is in a disordered state. Most likely it exhibits an amorphous-like structure, and coats the tungsten crystals uniformily with a constant thickness, thus forming a Ga skeleton structure. The non-crystalline gallium seems to undergo no first order structural phase transitions, e.g., no first order melting transition was noted when the composit was below, at or above the equilibrium melting point of Ga. The properties of gallium as grain boundary component differ significantly from those of crystalline and amorphous bulk gallium.
\end{abstract}

PACS: 61.10.-i, 65.70.+y, 72.80.Tn, 74.70.Ad

\section{INTRODUCTION}

Technical materials like, e.g., metals or ceramics, are often used as polycrstalline materials. Usually, the fraction of atoms within grain boundaries is many orders of magnitude smaller than the fraction of atoms within individual grains. In contrast, the invention of nanocrystalline materials in 19811 has yielded the possibility to synthesize solids with nm size grains so that the number of atoms located within grain boundaries and grains are comparable. This leads to an enhanced interface surfaceto-volume ratio, and hence mechanical, electrical, magnetical or thermal properties are affectedsignificantly by the atoms within the grain boundariest. 3 .

Beside generating nanocrystalline materials consisting of only one kind of atoms, it should be also possible to produce nanocrystalline materials in which the crystallites and the interfaces have different chemical compositions (e.g., A and B) [c.f. Fig. 1]. Materials suitable to generate nanocrystalline solids of this type have to meet the following two conditions. First, they should be unmiscible. Furthermore, the material used for the grain boundary component should wet the material used for the formation of the crystalline component. One way of synthesis is to produce small crystallites of $\mathrm{A}$ and coat them with B. By subsequently compacting the coated particles one obtains a composite consisting of nanometer-sized grains of A atoms, separated by a grain boundary component of B atoms. The new aspect of such skeleton structures is, that it can be expected that the $\mathrm{B}$ atoms within the grain boundaries are prevented to crystallize in their equilibrium structure because of the interatomic forces excerted by the adjacent crystallites of A atoms.

By investigating such a two-phase material, the major questions, we shall focus on, are:

(i) What kind of structure do the B atoms form within the grain boundaries?

(ii) Does the grain boundary component of B atoms undergo structural phase transitions upon heating/cooling? (iii) What can be learned about thermal excitations and electronic properties of the boundary component?

In the literature, some attempts are reported to synthesize such structures. Although some experimental results 6 cexist and are confirmed by theoretical considerations 0 , it yas not yet to manufacture pure skeleton structures 10 , where component B just forms the grain boundary component without larger agglomerates. The experiments and the model, with resembles the thermodynamics and the processing mechanism presented here are graphite encapsulated nanocrystalline particlest1. 12.

In this publication, we present measurements of structural, thermal, electrical and magnetic properties of $\mathrm{W}$ Ga composites. Peculiarities of physical properties observed for these samples will be discussed with respect to the gallium grain boundary component (gallium skeleton).

\section{EXPERIMENTAL}

Tungsten (W) as a high refractory metal and gallium $(\mathrm{Ga})$ as a low-melting metal $\left(\mathrm{T}_{m}=29^{\circ} \mathrm{C}\right)$ seem to be a suitable model system for the formation of twocomponent composites of the type described in Fig. 1. The wetting properties of Ga on W surfaces is well known from FIM experiment,13,14. Furthermore, the solubility of $\mathrm{W}$ in $\mathrm{Ga}$ ever at temperatures as high as $815^{\circ} \mathrm{C}$ is 0.001 to $0.008 \mathrm{wt} \% 15$. The nanocrystalline samples were $\mathrm{pro}$ duced by a modified inert gas condensation method 16,17 . The high refractory $\mathrm{W}$ was dc-sputtered from a $\mathrm{W}$ target (purity $99.99 \%$ ), while the Ga (purity 99.9999\%) was evaporated using a conventional evaporation source from a $\mathrm{W}$ boat. The inert gas atmosphere consisted of $\mathrm{Ar}\left(\mathrm{p}_{A r}\right.$ $\approx 7$ mbar). The experimental setup is shown in Fig. 2. 
A variation of the process parameters like, e.g., inert gas pressure, power or source-collector distapse can significantly influence the particle formation 16 . 17 . The formation of nanocrystalline $\mathrm{W}$ crystals takes place within the Ar atmosphere, while the evaporated Ga coats these crystallites subsequently. These crystallites are then transferred due to the gas convection between the hot source and the cold collecting device to a cooling finger, which is held at liquid nitrogen temperature. The nm-sized crystallites collected on the surface of the cooling finger were subsequently stripped off and were then compacted $\left(\mathrm{p}_{c}>1 \mathrm{GPa}\right)$ to macroscopic samples of typically $8 \mathrm{~mm}$ in diameter and thicknesses of 0.3 to $1 \mathrm{~mm}$ under UHV conditions. The thickness of the Ga layer depends on the Ga vapor pressure within the vacuum chamber. The Ga layer thicknesses were varied from less than a monolayer Ga up to five monolayers. The thicknesses of the layers were determined by measuring the overall amount of $\mathrm{Ga}$ within the samples as well as the grain size 18 .

\section{RESULTS AND DISCUSSION}

Pure nanocrystalline $\mathrm{W}$ specimen were synthesized as reference samples. For the pure $\mathrm{W}$ as well as for W-Ga samples, the grain sizes of the $\mathrm{W}$ crystallites were in the range of 8 to $13 \mathrm{~nm}$. Figure 3(a) shows the diffraction pattern (wide angle X-ray diffraction, XRD, $\mathrm{Cu} \mathrm{K}_{\alpha}$ radiation) of the reference sample, having a grain size of $\approx 13$ $\mathrm{nm}$. The diffraction pattern indicates that the samples do not have the equilibrium bcc structure of W19. In fact, the $\mathrm{W}$ crystallized in a metastablemodification, which is known as A15 or $\beta-\mathrm{W}$ structure 20 . Figure 3 (b) displays an XRD measurement of a nanocrystalline W-Ga sample with a Ga content of $\approx 35$ at $\%$. Even for Ga contents as high as 45 at\%, the XRD pattern displayed only the maxima which correspond to A15-W. Bragg peaks due to Ga crystals were not observed, indicating that little or none of the $\mathrm{Ga}$ incorporated within the samples is crystalline. Moreover, neither a Laue background nor additional broad maxima, which would be characteristic for a disordered or ordered W-Ga solid solution, could be detected. The structural analysis of the $\mathrm{W}$ crystallites in the W-Ga composite shows, that no Ga is incorporated within the $\mathrm{W}$ lattice, i.e., the lattice parameters as well as the amount of strain is unchanged with respect to pure nanocrystalline W-A15 samples (lattice parameter of the crystalline A15 lattice $5.05 \AA$ ). The amount of gaseous impurities determined by hot extraction was equal for all samples, i.e., $\mathrm{N}_{2} \approx 2$ at $\%, \mathrm{H}_{2} \approx 1.5$ at $\%$ and $\mathrm{O}_{2} \approx 2.5$ at $\%$.

Let us now address to the major question, where is the Ga located and what is the structure of the Ga? There seem to be three conceivable reasons, why no Ga diffraction effects were observed:

(a) The Ga atoms are agglomerated, forming very small particles of a second component, which cannot be de- tected by XRD measurements by means of line broadening.

(b) The Ga atoms coat the nanocrystalline $\mathrm{W}$ grains and form an epitactic layer, which also has an A15 structure like the $\mathrm{W}$ nanocrystals.

(c) The W-Ga composite represents an ideal system with respect to the case that the $\mathrm{W}-\mathrm{A} 15$ particles are the crystalline component, whereas the Ga forms a skeleton, which structure corresponds to none of the known Ga modifications. The Ga skeleton may show an amorphous X-ray diffraction signal, which probably cannot be distinguished from the W-diffraction effects because of the large difference in the electron densities for $\mathrm{Ga}$ and $\mathrm{W}$, respectively.

To clearify, which model should be adapted, thermal expansion, DSC, TEM, PAC, resistivity measurements as well as the magnetic behavior of W-Ga composites were investigated. Generally, if the thermal expansion of a nanocrystalline material consisting of one sort of atoms is explored, a deviation from the linear expansion at temperatures above room temperature has been observed21. This result is interpreted in terms of relaxation processes in the grain boundaries of the nanocrystalline materials. This type of reduction of the thermal expansion was also observed for pure nanocrystalline $\mathrm{W}$ A15 samples [c.f. Fig. 由(a)]. However, the thermal expansion of nanocrystalline W-Ga composites differs significantly: No relaxation above room temperature was noticed [c.f. Fig. A(b)]. This points to the fact that a fundamental difference between grain boundaries in singlecomponent nanocrystalline materials as, e.g., W and in nanocrystalline composits, exists. Furthermore, no volume changes due to the melting of Ga wa recorded if the W-Ga specimen were heated to temperatures above the equilibrium melting temperature of Ga.

The absence of a solid/liquid phase transition of Ga at the thermodynamic melting point was analyzed in more detail by means of DSC measurements. The measurements were performed with an isoperibolic calorimeter in the low-temperature regime $\left(15 \mathrm{~K}<\mathrm{T}_{\text {meas }}<200 \mathrm{~K}\right)$ and a standard DSC (Perkin-Elmer DSC 7) in the temperature range of $200 \mathrm{~K}<\mathrm{T}_{\text {meas }}<700 \mathrm{~K}$. In the entire temperature range of 15 up to $700 \mathrm{~K}$ no DSC signal of a solid/liquid phase transition or glass transition could be detected. Figure 5 (a) shows the thermal expansion and the DSC signalk of W-30 at\% Ga specimen in the temperature range of 210 to $370 \mathrm{~K}$. A reference sample of pure $\mathrm{Ga}$ of about the same amount of $\mathrm{Ga}$ as in a WGa composites [Fig. 5 (a)] revealed clearly the melting enthalpy of Ga (Fig. 5).

Furthermore, conventional transmission electron microscopy was implemented to analyze the samples with respect to $\mathrm{Ga}$ agglomerates. Irrespective of the sample preparation method (ion milling, microtom cutting or simply breaking), no Ga crystallites nor Ga agglomerates of a second phase were observed. The Ga atoms, which could be detected by EDX measurements during SEM and TEM analysis, remained invisible in dark/bright field 
TEM.

If all observations reported so far, are compared with the models discussed above, the idea of an epitactic Ga layer, which exhibits an A15 structure, as well as the model of an amorphous Ga layer appear to be consistent with the experiments.

An additional analysis of the structure of the Ga phase was performed by using the perturbated $\gamma$ - $\gamma$-angle correlation (PAC) technique, which has been applied to study the structure of cold- condensed Ga film.22. Samples containig about $30 \mathrm{at} \% \mathrm{Ga}$ were used for these investigations. ${ }^{111} \mathrm{Cd}$ was used as probe, which was introduced in the nanocrystalline W-Ga samples by diffusion. Temperature and time for this process were chosen such, that no structural changes occured according to XRD and the calorimetry measurements. For example, the grain diameter of the W-A15 crystallites remained unchanged. Figure 6 displays a PAC spectrum measured at a temperature of $280 \mathrm{~K}$ [Fig. 6 (a)] and the corresponding frequency analysis [Fig. 6(b), line]. The spectrum shows a shape which corresponds to a highly disturbed, noncrystalline Ga structure within the nanocrystalline WGa composites. For comparison, the frequency analysis of amorphous Ga [c.f. Fig. 6 (b), dotted line) in terms of a continious random packing model, measured at $\mathrm{T} \leq 15$ $\mathrm{K} 22$, is also displayed. In the entire temperature range of $77 \mathrm{~K} \leq \mathrm{T}_{\text {meas }} \leq 700 \mathrm{~K}$, the PAC signals were unaffected by temperature changes and look like Fig. 6 (a). Even at or above the equilibrium melting temperature of $\alpha$-Ga, the PAC signal showed no detectable variation. A variation was noticed, however, if grain growth occured or if the structure of the $\mathrm{W}$ crystallites was changed from A15 to bcc by means of annealing. These observations seem to indicate that the boundary conditions excerted by the $\mathrm{W}$ crystallites on the atoms that control the atomic arrangement of the $\mathrm{Ga}^{23}$.

In order to obtain further information on the Ga structure, additional experiments on the electrical and magnetic behavior of the samples were performed. Figure 7(b) shows a measurement of the specific electrical resistance of a pure $\mathrm{W}$-A15 sample with an average grain diameter of $10 \mathrm{~nm}$, measured by the four-point probe method. A high absolute value of the specific resistance and a positive temperature coefficient of resistivity (TRC) is noted. W-Ga samples with a Ga content of $\approx 30$ at $\%$ and a grain size of $10 \mathrm{~nm}$ (A15 structure) [Fig. [(b)] exhibited a significant difference compared to pure $\mathrm{W}$-A15 samples. The absolute resistivity value was lower than for pure $\mathrm{W}-\mathrm{A} 15$, and the TRC was negative. Hence, the TRC differs not only from the one of pure W-A15 [c.f. Fig. 月(a)], but also from the TRC of pure $\mathrm{Ga}$, which is positive as well in the crystalline as in the liquid state2427.

The changed TCR of a W-Ga composite (compared to a pure W-A15 sample with comparable grain size) may be interpreted by assuming that the current only flows primarily through the grain boundary phase of Ga. Therefore, the measured specific resistivities have to be normalized to the volume portion of Ga present in the sample. Figure 8(a) shows a schematics of the sample, whereas Fig. 8 (b)-(d) show measurements of the specific resistance of W-Ga composites for different Ga contents. In all cases, the grain diameter of the W-A15 nanocrystals is $\approx 10 \mathrm{~nm}$.

The data may be understood as follows. With increasing Ga content within the samples, which corresponds to an increasing thickness of Ga in between the W-A15 grains, the resistivity decreases from about $145 \mu \Omega \mathrm{cm}$ (for $\approx 26$ at\% Ga) to $94 \mu \Omega \mathrm{cm}$ (for $\approx 40$ at $\% \mathrm{Ga}$ ). Compared to these values, the resistivities of $\alpha-\mathrm{Ga}(\approx 53$ $\mu \Omega \mathrm{cm})$ and liquid $\mathrm{Ga}(\approx 26 \mu \Omega \mathrm{cm})$ are lower 28 . Several samples were investigated, all exhibiting a negative TRC. The appearance of a negative TRC of Ga is contrary to all observations up to now. Parallel to the decrease of the specific resistance with increasing Ga content, the slighly negative TRC decreases which, for samples with a Ga content of 40 at\%, leads to a nearly constant resistivity in the temperature range of 5 to $285 \mathrm{~K}$, fluctuating only $0.01 \mu \Omega \mathrm{cm}$ around its mean value of $\approx 94 \mu \Omega \mathrm{cm}$.

The steep drop of the specific resistance below $5 \mathrm{~K}$, which is indicated by the arrows in Figs. \&(b)-(d), was investigated in more detail with a Foner Magnetometer (VSM). If this drop is caused by the onset of superconductivity, VSM measurements should exhibit the diamagnetic behavior of a superconducting material. No Meissner-Ochsenfeld effect was revealed for pure nanocrystalline W-A15 samples and W-Ga composites $(\approx 35 \mathrm{at} \% \mathrm{Ga})$ in the temperature range of $3.8 \mathrm{~K} \leq$ $\mathrm{T}_{\text {meas }} \leq 300 \mathrm{~K}$ and $5 \mathrm{~K}<\mathrm{T}_{\text {meas }} \leq 300 \mathrm{~K}$, respectively. For temperatures lower than $5 \mathrm{~K}$, the W-Ga composite showed the behavior of a type-II superconductor [Fig. 9]. The onset of superconductivity for temperatures below 5 $\mathrm{K}$ lies between the tempeartures of the onset of superconductivity of crystalline $(1.07 \mathrm{~K})$ and of amorphous $(8.4 \mathrm{~K}) \mathrm{Ga}$. The onset of superconductivity at $5 \mathrm{~K}$ may originate from proximity effects or from the presence of a new Ga structure within the composites. The considerable hysteresis (Fig. 9) points to a strong flux pinning in a highly disturbed material29. An estimation 30 of the Ginzburg-Landau parameter $\kappa$ of the sample shown in Fig. 9 yields $\kappa \approx 30$. This high value implies, that this sample is a strong type-II superconductor with a strongly reduced mean free path. For comparison, the mean free path in crystalline bulk Ga, as large as $2 \mathrm{~mm}$ at $4.2 \mathrm{~K} 31$. The W-Ga composit has kept its superconductivity below $5 \mathrm{~K}$, whether superconductive properties were not affected by heating the W-Ga composits above the melting point of bulk crystalline $\left(\mathrm{T}_{m}{ }^{G} a\right)$ Ga. This result seems to agree with the DSC measurements reported preeviously: the DSC measurements revealed no phase transition at $\mathrm{T}_{m}{ }^{G} a$. Apparently, the atomic arrangement formed by the $\mathrm{Ga}$ atoms in the grain boundaries is fixed by the boundary conditions, which are determined by the adjacent nanocrystalline $\mathrm{W}$ crystallites. 


\section{CONCLUSIONS}

W-Ga composites seem to be a suitable system with respect to the production of two-component composites, where the nanometer-sized grains of a first component (e.g., W) are coated by material of a second boundary component (e.g., Ga). The compacted composites may be regarded as a material, having a skeleton structure, whereby the skeleton is formed by the grain boundary component. Structure and stability of the boundary component is dominated by the nanometer-sized crystallites. The results reported above indicate, that the Ga grain boundary component has an atomic structure which seems to differ from the crystalline as well as from the amorphous state of Ga. This result seems to suggest that the boundary conditions excerted by the W-A15 nanocrystals on the Ga atoms determine the Ga structure. These boundary conditions appear to control the properties of the Ga boundary component. In the entire temperature range $(5 \ldots 700 \mathrm{~K})$ studied, the grain boundary component neither exhibited a glass transition nor was melting detectable.

It could be shown that the synthesis of W-Ga composites leads to samples, which physical properties are strongly determined by the grain boundary skeleton. It appears likely, that other alloy systems which fulfill the conditions unmiscible and wetting, are suitable for synthesizing nanocrytsalline composite materials consisting of a similar skeleton structure, as revealed in W-Ga. In fact, a structure of this type was recently reporrted for nanocrystalline $\mathrm{Al}_{2} \mathrm{O}_{3} / \mathrm{Ga}$ composits 10 . It is expected that such skeleton structures are interesting materials with respect to their magnetic behavior, i.e., magnetization, magneto-caloric effect or GMR.

\section{ACKNOWLEDGMENTS}

The authors would like to thank H. Wolf and Th. Wichert for performing PAC measurements.

${ }^{1}$ H. Gleiter, in: Deformation of Polycrystals: Mechanism and Microstructures, Proc. of the 2nd Ris Int. Symp. on Metallurgy and Mat. Sci., M. Hansen, A. Horsewell, T. Leffers and H. Lilholt (eds.), p. 15, Verlag (1981).

${ }^{2}$ R.W. Siegel, MRS Bulletin XV(10), 60 (1990).

${ }^{3}$ H. Gleiter, Nano94 - Second International Conference on Nanostructured Materials, Stuttgart (1990).

${ }^{4}$ J.S.C. Jang and C.C. Koch, J. Mater. Res. 5, 325 (1990).

${ }^{5}$ T. Ogura, C.J. McMahon, H.C. Feng, and V. Vitek, Acta Metall. 26, 1317 (1978).

${ }^{6}$ T. Haubold, F. Boscherini, S. Pascarelli, S. Mobilio, and H. Gleiter, Phil. Mag. 66, 591 (1992).
${ }^{7}$ D. Turnbull, J.S.C. Jang, and C.C. Koch, J. Mater. Res. 5, 1731 (1990).

${ }^{8}$ M. Yan, M. Sob, D.E. Luzzi, V. Vitek, G.J. Ackland, M. Methfessel, and C.O. Rodriguez, Phys. Rev. B47, 5571 (1993).

${ }^{9}$ P. Boolchand and C.C. Koch, J. Mater. Res. 7, 2876 (1992).

${ }^{10}$ H. Konrad, C. Karmonik, J. Weissmüller, H. Gleiter, R. Birringer, and R. Hempelmann, Physica B234-236, 173 (1997).

11 J.J. Horst, J.A. Block, K. Parvin, V.P. Dravid, J.L. Alpers, T. Sezen, and R. La Duca, J. Appl. Phys. 83, 793 (1998).

12 B.R. Elliot, J.J. Horst, V.P. Dravid, M.H. Teng, and J.H. Hweng, J. Mat. Res. 12, 3328 (1998).

13 O. Nishikawa and T. Utsumi, J. Appl. Phys. 44, 955 (1973).

${ }^{14}$ M. Konishi, M. Wada and O. Nishikawa, Surf. Sci. 108, L463 (1981).

15 T.B. Massalski (ed.), Binary Alloy Phase Diagrams, American Society for Metals (Metals Park, Ohio, 1986).

${ }^{16}$ H. Hahn and R.S. Averback, J. Appl. Phys. 67, 1113 (1990).

17 V. Haas and R. Birringer, Nanostruct. Mat. 11, 491 (1992).

18 J. Weissmüller, Nanostruct. Mater. 3, 261 (1993).

${ }^{19}$ W. Krauss and R. Birringer, Nanostruct. Mat. 9, 109 (1997).

${ }^{20}$ L. Cortella, B. Vinet, P.J. Desré, A. Pasturel, A.T. Paxton, and M. van Schilfgaarde, Phys. Rev. Lett. 70, 1469 (1993).

${ }^{21}$ M. Klingel, PhD thesis, University of Saarland (1992).

${ }^{22}$ P. Heubes, D. Korn, G. Schatz and G. Ziebold, Phys. Lett. 74A, 267 (1979).

${ }^{23}$ H. Wolf, H.G. Zimmer, T. Filz and Th Wichert, Nano94 Second International Conference on Nanostructured Materials (Stuttgart, 1994).

${ }^{24}$ E.I. Geshko, V.P. Mikhal'chenko and B.M. Sharlai, Sov. Phys. Solid State 14, 1554 (1972).

${ }^{25}$ M. Yaqub, D. Waldorf, R. Boughton and W.A. Jeffers, Phys. Lett. 23, 423 (1966).

${ }^{26}$ W. Buckel and W. Gey, Z. Phys. 176, 337 (1963).

${ }^{27}$ G. Ginter, J.G. Gasser and R. Kleim, Phil. Mag. B54, 543 (1986).

${ }^{28}$ Kirk-Othmer, Encyclopedia of Cemical Technology 11 (3rd ed.) (1980), p. 606.

${ }^{29}$ W. Buckel, Supraleitung (Physik-Verlag, 1984), p.164.

${ }^{30}$ A. Campell, J.E. Evetts and D. DewHughes, Phil. Mag. 10, 333 (1964).

31 J.F. Cochran and M. Yaqub, Phys. Rev. 140A, 2174 (1965).

FIG. 1. Schematics of a nanocrystalline material composed of a component A, representing the grains (open circles) and a component B, representing the grain boundary component (black circles).

FIG. 2. Schematics of the vacuum chamber used for the inert gas condensation method, containing a tungsten sputter source and an evaporation source for Ga. 
FIG. 3. (a) Diffraction pattern of a nanocrystalline $\mathrm{W}$-A15 sample. The grain diameter is $\approx 13 \mathrm{~nm}$. (b) Diffraction pattern of a nanocrystalline $\mathrm{W}$-Ga sample. The Ga content is 40 at\%. All maxima correspond to W-A15 with a grain diameter of $\approx 8 \mathrm{~nm}$. No Bragg peaks corresponding to crystalline Ga could be detected.

FIG. 4. Thermal expansion during heating of a W-Ga composite $(\mathrm{W}$ grain diameter $\approx 10 \mathrm{~nm}, \mathrm{Ga}$ content $\approx 30 \mathrm{at} \%$ ) (a) in comparison of a pure $\mathrm{W}$ sample (grain diameter $\approx 10$ $\mathrm{nm}$ ) (b) for temperatures between 130 and $350 \mathrm{~K}$.

FIG. 5. (a) DSC signal of a W-Ga composite (30 at\% $\mathrm{Ga}$ ). The melting point of pure $\alpha-\mathrm{Ga}$ is $303 \mathrm{~K}$. Neither at this temperature nor at the melting points of other modifications of $\mathrm{Ga}$ an endothermic signal is detected. (b) DSC signal of the same amount of pure Ga like in (a).

FIG. 6. (a) PAC spectrum of ${ }^{111} \mathrm{Cd}$ in a nanocrystalline W-Ga composite (Ga content $\approx 30$ at $\%$ ) at a temperature 280 K. (b) Frequency analysis of the PAC spectrum of (a). For comparison, the frequency analysis of amorphous Ga (shaded) according to Heubes et al 22 ( $\mathrm{T} \leq 15 \mathrm{~K})$ is shown.

FIG. 7. (a) Specific resistance of a nanocrystalline W-A15 sample as a function of the temperature. The grain diameter is $\approx 10 \mathrm{~nm}$. (b) Specific resistance of a nanocrystalline W-Ga composite $(<30$ at\% $\mathrm{Ga})$ as a function of the temperature. The grain diameter of $\mathrm{W}$ is $\approx 10 \mathrm{~nm}$.

FIG. 8. (a) Model of a nanocrystalline W sample (left side) and of the Ga skeleton structure (right side). (b) - (d) Resistivities of the W-Ga composite as a function of the temperature and varying $\mathrm{Ga}$ content. In the normal conductive state the TRC is a function of thickness $(\approx$ zero for about 40 at\% Ga). The Ga content within the nanocrystalline $\mathrm{W}$ samples is (b) W $<30$ at\% Ga, (c) W/33 at\% Ga, and (d) $\mathrm{W} / 40$ at\% Ga.

FIG. 9. Magnetic behavior of a W-Ga composite (mean grain diameter of $\mathrm{W} \approx 8 \mathrm{~nm}$, Ga content $\approx 35$ at $\%$ ) at $\mathrm{T}<$ $5 \mathrm{~K}$ in a magnetic field. 
Fig.1 Krauss et al., Skeleton structures...

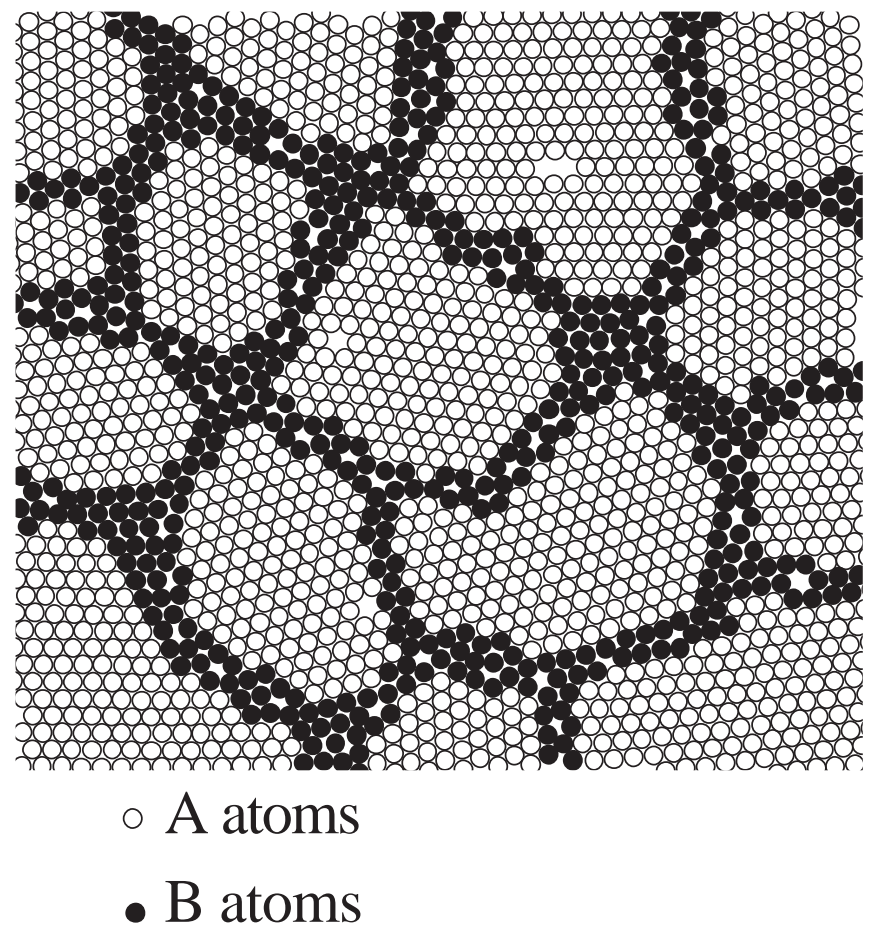


Fig.2 Krauss et al., Skeleton structures...

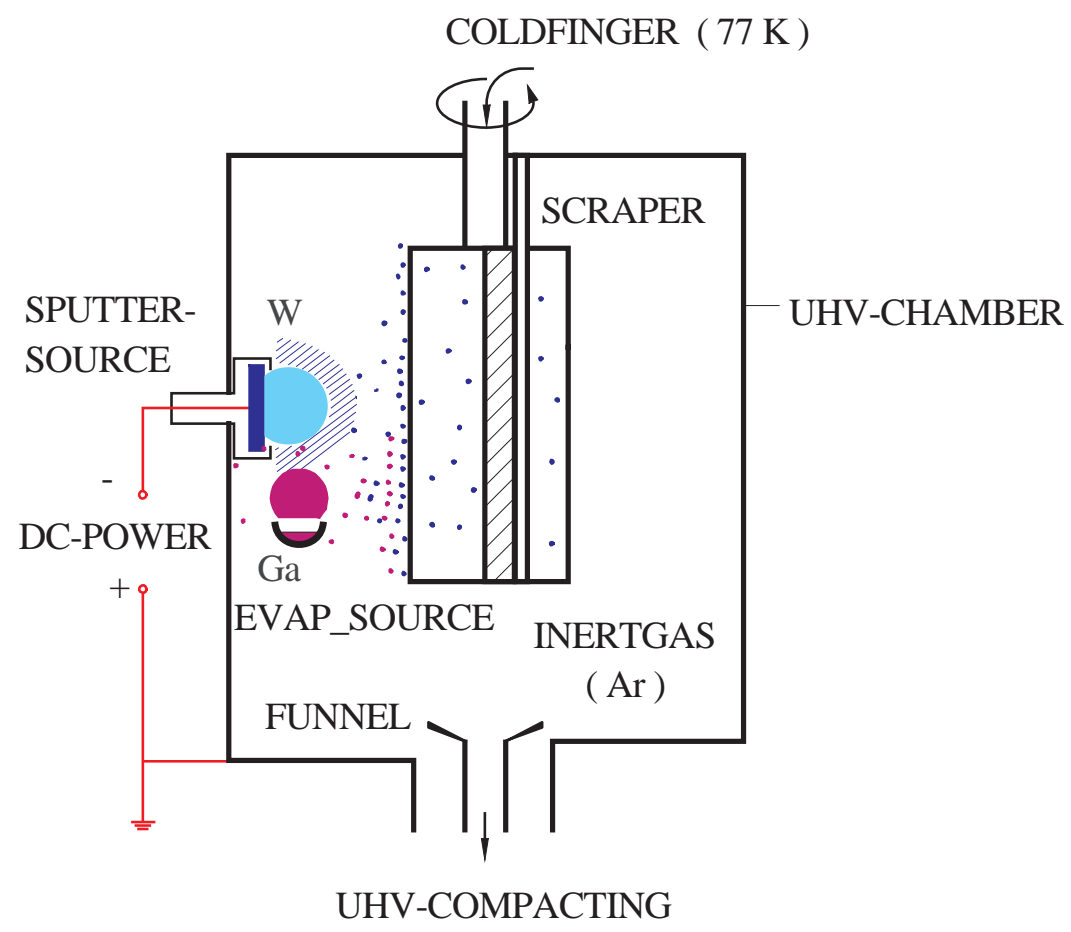

